\title{
Epigenetic Mechanism of TGF- $\beta 1$ Promoting Osteogenic Differentiation of Human Bone Marrow Mesenchymal Stem Cells in Osteoarthritis
}

Jianwei He ( $\sim$ hjw5566@126.com )

The First Affiliated Hospital of Shihezi university, school of medicine https://orcid.org/0000-00030628-4182

\section{Weiwei Cao}

key laboratory of Xinjiang Endemic and Ethnic Disease, Shihezi universtiy, school of medicine

\section{Qinzheng Fang}

Department of orthopaedics of Shihezi university, school of medicine

\section{Inayat Azeem}

Office for Education to international students, school of medicine, shihezi university

Wei Liu

Key Laboratory of Xinjiang Endemic and Ethnic disease, shihezi universtiy, school of medicine

\section{Research Article}

Keywords: human bone marrow mesenchymal stem cells, RUNX2, epigenetics, Transforming growth factor $\beta 1$, Methyltransferases, demethylase

Posted Date: July 30th, 2021

DOl: https://doi.org/10.21203/rs.3.rs-732375/v1

License: (9) (1) This work is licensed under a Creative Commons Attribution 4.0 International License. Read Full License 


\section{Abstract}

Objectives: It had been proved that TGF- $\beta 1$ was correlated with onset of osteoarthritis in vitro and vivo. Here, This study was to elucidate the epigenetic mechanism of TGF- $\beta 1$ promoting osteogenic differentiation in osteoarthritis.

Methods: hBMSCs surface antigens were assayed by flow cytometry tests. qRT-PCR was performed to detect hBMSCs mRNA levels of RUNX2, PPARY and SOX9. hBMSCs were stained by osteoalkaline phosphatase and alizarin red. The qRT-PCR and Western blot were both used to detect the expression levels of methylases, demethylases and osteogenic transcription factor RUNX2 after hBMSCs were cultrued in osteogenic medium coincubated with TGF- $\beta 1$ solution.

Results: hBMSCs were identified by over expressions of CD90, CD105 and CD44, as well as the positive multi-diffenentiation potential tests. hBMSCs bone alkaline phosphatase and alizarin red staining were observed to deepen in TGF- $\beta 1$ group compared with the osteogenic culture group. The mRNA expression levels of $E Z H 1, K D M 2 B, K D M 4 A / 4 B / 4 C / 4 D$, and KDM6A /6B were increased in hBMSCs cultured in osteogenic medium. The expression levels of $\mathrm{KDM} 6 \mathrm{~A} / 6 \mathrm{~B}$ were shown increasement when TGF- $\beta 1$ was co-incubated with osteogenic medium. Furthermore, the mRNA and protein levels of KDM6A/6B were significantly decreased after SB431542 was added in the medium. RUNX2 was significantly inhibited by the addition of GSK-J4 solution, while KDM6A/6B expression level did not change significantly.

Conclusion: The osteogenic differentiation of hBMSCs was related to the enhanced expressions of EZH1, KDM2B, KDM4A-4D, KDM6A/6B. The expression levels of demethylase KDM6A/6B were positively regulated by the TGF- $\beta / S m a d$ signaling pathway, which promoted the osteogenic differentiation of hBMSCs.

\section{Background}

It was reported that there were more than 27 million Knee Oosteoarthritis (KOA) patients in the United States in 2009[1], estimated to exceed 50 million people in 2020 [2], the incidence of KOA in China was as high as $8.1 \%$ in 2016 [3], and the incidence in people over 60 years old was even more than $30 \%$ [4]. At present, the molecular mechanism of the KOA onset is still unclear, and there is still lack of effective treatment to inhibit the KOA occurrence and progress of OA [5].

Liang et al proposed the hyperthesis that the occurrence of KOA was caused by abnormally increased TGF- $\beta 1$ in the subchondral bone, which was proved in vitro and animal models. When the joint was subjected to abnormal mechanical and mechanical stimulation, the osteoclast bone resorption enhanced in the subchondral bone, and a large amount of TGF- $\beta 1$ stored in the bone matrix was activated, which disturbed the subchondral bone internal environment. Bone marrow mesenchymal stem cells (BMSCs) were induced by the abnormally increased concentration of TGF- $\beta 1$ to ectopic osteogenesis in subchondral bone, leading to the increased formations of bone islets and sclerosis. Then the $\mathrm{OA}$ pathological changes occurred[6]. Abnormal osteogenesis of BMSCs was characterized by bone spur 
formation and subchondral bone sclerosis, which was also the main clinical feature of OA in X-ray and CT examinations [7]. OA joint pain was also a clinical symptom caused by bone marrow injury in subchondral bone sclerosis, which could also predict the severity of OA condition [8]. The application of TGF- $\beta 1$ inhibitors could significantly reduce the number of bone formation, confirmed by MRI imaging data in orthopedic clinical patients[9].

It could be seen that hBMSCs ectopic osteogenesis induced by TGF- $\beta 1$ was a key factor in the occurrence and development of OA. Therefore, it was necessary to study the molecular mechanism of TGF- $\beta 1$ promoting the osteogenic differentiation of hBMSCs.

The pathogenic mechanism of epigenetics is to change the expression of genes without the alteration of the main genes DNA sequence [10]. In eukaryotes, there were three types of epigenetic mechanisms at the same time, DNA chemical modification, histone modification and non-encoding regulatory RNAs [11], respectively. Histones modification could regulate gene expression through methylation, acetylation and many other reversible chemical modifications[12]. The histone methylation modification site was located on the lysine or arginine residues of the third or fourth subunit of histone. Histone lysine methyltransferases (Histone lysine Methyltransferases, HMTs) and demethylases (Histone lysine demethylases, KDMs) were two kinds of enzymes that catalyzed the methylation modification of histones. The role of HMTs was to trimethylate single, double or lysine residues at different positions on the third subunit of histones. The role of KDMs was to remove the methyl groups on lysine residues, thereby activating or inactivating genes. A variety of histone lysine methyltransferases had been discovered $\triangle \mathrm{D} O \mathrm{OT} 1 \mathrm{~L}[13], \mathrm{EZH} 2, \mathrm{EZH1}$ [14]. Many kinds of demethylation transferases had also been found, LSD1[15], KDM2A-2D, KDM3A-3D, KDM4A-4D, KDM5A-D, KDM6A, KDM6B[16] and so on.

Previous studies confirmed that the lysine residues on the promoters of genes were affected by methyltransferase and demethylase, such as bone formation-related transcription factor RUNX2 and cartilage-related transcription factor SOX9. The methylation and demethylation reactions were adjusted, thereby regulating the expressions of RUNX2 and SOX9 [17], ultimately led to the onset of OA. Previous studies showed KDM6B indirectly regulated RUNX2 gene expression through regulation of BMP and HOX gene expression in BMCs osteogenesis differentiation [18]. It was reported that KDM4B was required for the cartilage differentiation of hBMSCs mediated by the TGF- $\beta 1 / S m a d 3$ signaling pathway[19]. Anna Cascante reported that $\mathrm{KDM} 4 \mathrm{~A} / 4 \mathrm{C}$ activated the stem cells differentiation in vivo and vitro[20]. KDM2A[21] and KDM2B[22] were proved to promote hBMSCs differentiation into dentin osteogenesis. Ezh2 was also confirmed to inhibit the adipogenic differentiation and enhance the osteogenesis of stem cells [23].

So we asked questions as following:

What methyltransferase and demethylase expression levels changed in the process of hBMSCs osteogenesis? Were the changed expression levles of methyltransferases or demethylases related with the TGF- $\beta 1 /$ Smad3 signaling pathway? 
To solve the first problems, the mRNA and protein expression levels of methyltransferases and demethylases were both detected to show the alterative expression levels of these enzymes in hBMSCs cultured in osteogenic medium, compared with that in regular medium.

To solve the second question, TGF- $\beta 1 /$ Smad3 signaling pathway inhibitors were needed to use to specifically inhibit the signaling pathway activity in osteogenic differentiation of hBMSCs, followed by detecting the expression levels of histone lysine demethylases and methyltransferases, which would explore how the expressions of demethylases and methyltransferases were associated with TGF$\beta 1 /$ Smad3 signaling pathway. Furthermore, the inhibitors of these demethylases were used to find out if demethylases were related with the expression level of RUNX2, which elucidated the epigenetic molecular mechanism of TGF- $\beta 1$ promoting bone differentiation of hBMSCs.

\section{Materials And Methods}

Cell culture: The bone marrow tissue was taken from a patient with hip joint OA who underwent total hip arthroplasty. 5-10 mL of bone marrow fluid was taken after reaming. After pre-wetting with a $50 \mathrm{~mL}$ disposable syringe with heparin injection, $5 \mathrm{~mL}-10 \mathrm{~mL}$ human bone marrow fluid was mixedwith equal amount DMEM, then centrifuged at $1000 \mathrm{rpm}$ for $5 \mathrm{~min}$, and the precipitation was collected. An equal volume of Percoll separation solution was added along the tube wall, and centrifuged at $2500 \mathrm{rpm}$ for 25 min. The middle layer was carefully sucked (the white film layer which was visible to naked eyes).. The collected monocytes were washed twice with PBS, then resuspended in DMEM (containing 10\% FBS), and $1 \times 10^{6} / \mathrm{mL}$ cells were inoculated into a culture flask $\left(25 \mathrm{~cm}^{2}\right)$, which was recorded as P0. The regular stem cell culture medium preheated to $37^{\circ} \mathrm{C}$ was added to the culture flask, then transferred to $5 \% \mathrm{CO}_{2}$, $37^{\circ} \mathrm{C}$ incubator to keep a static culture.

Some cell colonies were formed in 3 days, and the confluency density of the hBMSCs reached about $80 \%$ in 8 days, then the cells would be passaged and divided into 3 new flask $\left(75 \mathrm{~cm}^{2}\right)$.

Flow cytometry Detection First was fluorescent antibody labeling: the P3 generation cells were taken out, and $1 \times 10^{5}$ cells was prepared in each EP tube. Specific fluorescently labeled antibody $10 \mathrm{uL}$ was added into the EP tubes. There were CD 45 antibody (BD 555485), CD44 antibody (BioLegend 103011), CD90 antibody (BD 555595), CD34 antibody (BD 555821), CD105 antibody (BD 560839). The EP tubes were incubated at $4^{\circ} \mathrm{C}$ for $15-30 \mathrm{~min}$ in the dark. PBS cell suspensions were washed for 3 times, centrifuged at $1000 \mathrm{rpm}$, resuspended in 500 uL PBS in each EP tube. hBMSCs surface antigens were detected by Flow Cytometer tests.

qRT-PCR detection. RUNX2, PPARY, SOX9 mRNA expression in hBMSCs were detected by the quantitive real-time RT-PCR amplification (qRT-PCR). The conditions for amplifications were $55^{\circ} \mathrm{C}$ for $10 \mathrm{~min}, 95^{\circ} \mathrm{C}$ for $5 \mathrm{~min}$, followed by 36 cycles of $95^{\circ} \mathrm{C}$ for 5 seconds and $60^{\circ} \mathrm{C}$ for 10 seconds according to the instructions from the Rotor-Gene ${ }^{\circledR}$ SYBR ${ }^{\circledR}$ Green Handbook (Rotor-Gene SYBR Green RT-PCR Kit, 
Germany). Primers were designed by Primer5.0 design primer, and synthesized by BGI gene company. Primers were sequenced as following. (see Table1)

After the reaction, we obtained the original copy number of the cDNA products and the relative expression levels of mRNA in RUNX2,PPARY and SOX9. The experiments were repeated for 3 times with 3 replicate holes each time.The mRNA expression levels of methyltransferases and demethylases enzymes in hBMSCs were tested in a semi-quantitative test of qRT-PCR, and the primers devised by Primer 5.0 design primer were listed as followings.(see Table1)

Western blot: Cellular proteins were prepared using cell lysis buffer $(50 \mathrm{mM}$ Tris- $\mathrm{HCl}, \mathrm{pH} \mathrm{8.0,1 \%} \mathrm{Nonidet}$ P-40, $2 \mathrm{mM}$ ethylenediaminetetraacetic acid, $10 \mathrm{mM} \mathrm{NaCl}, 2 \mathrm{mg} / \mathrm{mL}$ aprotinin, $5 \mathrm{mg} / \mathrm{mL}$ leupeptin, $2 \mathrm{mg} / \mathrm{ml}$ pepstatin, $1 \mathrm{mM}$ dithiothreitol, $0.1 \%$ sodium dodecyl sulfate, and $1 \mathrm{mM}$ phenylmethylsulfonyl fluoride). The BCA method was used for total protein quantification. Equal amounts of protein samples $(8 \mu \mathrm{g})$ were separated by polypropylene gel electrophoresis with $5 \%$ concentrated gel and $12 \%$ separating gel under the stable voltage of 80 for 60 minutes, and then transferred to a PVDF membrane with the conditions of a stable 25 voltage 120 minutes for KDM6A, KDM6B, KDM4A, KDM4D, EZH1 and RUNX2 protein expressions.

PVDF membranes were all incubated by the solution prepared by skimmed milk powder, dissolved in Tris Buffered Saline Tween (TBST buffer) for 2 hours. Then the different diluted first antibody was added and incubated overnight at 4 degrees Celsius. The first antibody dilution ratioes were marked as Table 2.

This procedure was followed by incubation with sheep anti-rabbit $(1: 20,000)$ horseradish peroxidaselabeled secondary antibodies for $2 \mathrm{~h}$ at room temperature, whilst $\beta$-actin immunoblots served as loading controls. The membrane was photographic, developed and fixed, and an ECL reagent kit (Thermo Systems) was used to detect the bands on the membrane. Finally, the data and the images were collected by the Gel Imaging System (Bio-Rad, America) and X-ray exposure.

Bone alkaline phosphatase staining: After 6 days of osteogenic induction, the BMSCs cells were exchanged and soaked in PBS 3 times, 5 min each time, fixed with 4\% paraformaldehyde 400uL per well at room temperature for $30 \mathrm{~min}$, then soaked in PBS 3 times, for $5 \mathrm{~min}$ each time. According to the BCIP/NBT alkaline phosphatase staining kit instructions (Beyotime Co., Ltd), the cells were dyed and observed under a microscope, selecting the field of view to collect the images.

Alizarin Red Staining: After 14 days of osteogenic induction and culture, the hBMSCs cells were stained with Alizarin Red (Solarbio company, China). The procedures were followed in detail as below: hBMSCs cells were cultured in 6-well plates, and the cell fluid was changed and washed twice with PBS. The second was fixation. $4 \%$ paraformaldehyde fixation for $15 \mathrm{~min}$, paraformaldehyde was discarded, and the cells were washed with PBS for 3 times. The third was that $0.2 \%$ Alizarin Red staining solution $2 \mathrm{~mL}$ was added to each well and left alone for $30 \mathrm{~min}$. The fourth step was rinse. Alizarin Red dye solution was discarded and the cells in 6-well plates with PBS were rinsed for 6 times. Finally, the dyed cells were observed and pictures were taken under a microscope. 


\section{Results}

1. Isolation, culture and identification of hBMSCs.

1.1 Regular stem cell culture medium was used to inoculate and culture hBMSCs for 48 hours. Observed under an inverted microscope, hBMSCs showed a round shape, and most of them had adhered to the wall. After 4 days, the cells were spindle-shaped.At about 7 days, the cell colony gradually increased, and the confluence was $70-80 \%$.The morphology of the hBMSCs was shown in Fig.1.

1.2 Identification of hBMSCs antigen expression by flow cytometry. Flow cytometry results showed positive rates of antigen expressions including CD44, CD90, CD105, CD45, and CD34 were 99.7\%, 99.6\%, 99.4\%, 2.0\%, and 1.5\%, respectively, As shown in Fig. 1.

1.3 Detection of hBMSCs multi-diffenentiation potential tests. hBMSCs were cultured in osteogenic medium, adipogenic culture medium and chondrogenic medium, respectively. After 6 days, hBMSCs were collected and subjected to qRT-PCR to detect the mRNA expressions of RUNX2, PPARY and SOX9, marker genes of bone differentiation, adipogenic differentiation and chondrogenic differentiation. They were compared with the control group (the hBMSCs were cultured in regular stem cell culture medium) see Fig.1.

2. Alkaline Phosphatase and Alizarin Red staining experiments observation of TGF- $\beta 1$ 's promotion effect on the bone differentiation of hBMSCs

2.1 Alkaline Phosphatase staining. In the osteogenic culture group, A small amount of cell cytoplasm was found to be stained, indicating that a small amount of osteoblasts were formed. The results of TGF$\beta 1$ group (addition of osteogenic medium and TGF- $\beta 1$ final concentration $10 \mathrm{ng} / \mathrm{mL}$ ) showed that the cell cytoplasm staining darkened significantly, which denoted hBMSCs osteogenesis enhanced. See Fig. 2

2.2 Alizarin Red staining. After 14 days of culture in the osteogenic culture group, the hBMSCs were stained with Alizarin Red. After 14 days of culture in the TGF- $\beta$ group, the staining of the cells in the osteogenic culture group was significantly increased and the color was darkened, suggesting that the hBMSCs in the TGF- $\beta$ group osteogenesis enhanced. See Fig. 2.

3. Study on the epigenetic mechanism of TGF- $\beta 1$ ' promotion effect on the bone differentiation of hBMSCs.

3.1 The expressions of some methyltransferase and demethylase mRNA genes changed after the osteogenic differentiation of hBMSCs.

Compared with the hBMSCs cultured in regular culture medium, the demethylase KDM2B, KDM4A, KDM4B, KDM4C, KDM4D, KDM6A, KDMA6B and methyltransferase EZH1 mRNA genes were significantly increased in hBMSCs cultured by osteogenic medium $(P<0.05)$. There was no statistical difference in the mRNA expression of the demethylase KDM2A and the methyltransferase EZH2 $(P>0.05)$, which denoted 
that KDM2B, KDM4A, KDM4B, KDM4C, KDM4D, KDM6A, KDMA6B and EZH1 were related to the osteogenic differentiation of hBMSCs. The methyltransferase EZH2 and the demethylase KDM2A have no correlation with the osteogenic differentiation of hBMSCs. See Fig.3.

3.2 The expressions of methyltransferases and demethylases were partly regulated by TGF- $\beta /$ Smad signaling pathway.

3.21 mRNA expression levels of methyltransferase and demethylase were regulated by TGF- $\beta 1$. The hBMSCs were divided into the osteogenic culture group (osteogenic medium culture), the TGF- $\beta 1$ group (osteogenic medium and TGF- $\beta 1$ 10ng/mL) and the SB group (osteogenic medium and TGF- $\beta 110 \mathrm{ng} / \mathrm{mL}$ added with SB431542 1uM). SB431542 is a specific blocker of the TGF- $\beta /$ Smad signaling pathway. After 72 hours of incubation in each group, qRT-PCR was used to detect the mRNA expression of the target genes in the hBMSCs of each group.

Compared with the osteogenic culture group, the mRNA expression of the demethylases KDM2B, KDM4B and KDM4C in the TGF- $\beta 1$ group had no statistically significant difference ( $P>0.05)$, suggesting that expressions of the above three methylases were not associated with TGF- $\beta 1$ during the osteogenic differentiation of hBMSCs. The relative mRNA expressions of KDM4A, KDM4D and EZH1 were significantly reduced $(P<0.01)$, which denoted that the mRNA expressions of KDM4A, KDM4D and EZH1 were negatively regulated by TGF- $\beta 1$. The relative expressions of KDM6A and KDM6B mRNA in the TGF$\beta 1$ group were significantly increased by 1.5 times $(P<0.01)$, which showed that the mRNA expressions of KDM6A and KDM6B were both positively regulated by TGF- $\beta 1$. See Fig. 4.

3.22 Protein expression levels of methyltransferase and demethylase were regulated by TGF- $\beta 1$.

To confirm the regulatory effect of TGF- $\beta 1$ on the protein expressions of methyltransferase and demethylase, hBMSCs were grouped into the regular culture group, the osteogenic culture group, the TGF$\beta 1$ group and the SB group. The grouping methods were the same as the contents of 3.1 and 3.21. A Western Blot was used to detect the protein expressions of methyltransferase $E Z H 1$ and demethylase KDM4A, KDM4D, KDM6A and KDM6B in the above 4 groups of hBMSCs. The results showed that during the osteogenic differentiation of hBMSCs, the protein expression trends of methyltransferase and demethylase were the same with mRNA expressions of these enzymes. See Fig. 4.

3.3 Osteogenic transcription factor RUNX2 was regulated by KDM6A and KDM6B, which elucidated the mechanism of TGF- $\beta 1$ promoting the hBMSCs osteogenic differentiation.

The hBMSCs were divided into the regular culture group, the osteogenic culture group, the TGF- $\beta 1$ group and the GSK-J4 group (osteogenic medium and TGF- $\beta 1$ ( $10 \mathrm{ng} / \mathrm{mL}$ ) and GSK (10uM)). After 72 hours of incubation in each group, qRT-PCR was performed to detect the mRNA expressions of KDM6A, KDM6B and RUNX2. A Western Blot was used to detect the protein levels of KDM6A, KDM6B and RUNX2 in hBMSCs. See Fig. 5. 
Comparing the osteogenic culture group with the regular culture group, the relative expressions of $\mathrm{mRNA}$ and proteins of KDM6A, KDM6B and RUNX2 in the osteogenic culture group were significantly increased $(P<0.01)$, suggesting that the expressions of KDM6A and KDM6B were significantly related to the osteogenic differentiation of hBMSCs.

Compared with the osteogenic culture group, the relative expressions of mRNA and proteins of KDM6A, KDM6B, and RUNX2 in the TGF- $\beta 1$ group were furtherly increased $(P<0.01)$, which denoted TGF- $\beta 1$ had a positive regulatory effect on the expression of KDM6A, KDM6B and RUNX2.

Compoared with the TGF- $\beta 1$ group, it showed that the relative expressions of mRNA and proteins of KDM6A and KDM6B in the GSK-J4 group did not change significantly $(P>0.05)$ while the expressions of RUNX2 reduced significantly $(P<0.01)$, which indicated that osteogenic transcription factor RUNX2 was regulatd by the demethylases KDM6A and KDM6B. GSK-J4 was the specific inhibitor of KDM6A /6B activity. See Fig.5.

\section{Discussion}

According to the definition and requirements of International Society for Cell Therapy (2006 Edition) [24], hBMSCs are positive for various cell furface antigens such as CD29, CD44, CD71, CD90 and CD106, and negative for angiogenesis-related -antigens such as CD45 and CD34. The differentiation potential of BMSCs in vivo and in vitro was the most important feature that distinguished BMSCs from various differentiated adult cells. BMSCs isolated and cultured in vitro could undergo adipogenic, osteogenic and chondrogenic differentiation under the induction of special medium [25]. After the hBMSCs were cultured in osteogenic, adipogenic and chondrogenic medium, the mRNA expressions of osteogenic transcription factor Runx2, chondrogenic transcription factor Sox9, and adipogenic transcription factor PPARy were significantly increased, suggesting that the cultured cells had multi differentiation potentials.

After observing and verifying the effect of TGF- $\beta 1$ on the osteogenic differentiation of hBMSCs by alkaline phosphatase staining and alizarin red staining, we screened several methyltransferases and demethylases related to the osteogenic differentiation of hBMSCs by qRT-PCR. We also found that the levels of demethylases KDM4A, KDM4D and methyltransferase EZH1 were all significantly inhibited after TGF- $\beta 1$ was added into the osteogenic medium, suggesting that TGF- $\beta 1$ might promote the hBMSCs osteogenic differentiation by inhibiting the expression of these enzymes. Wang $Y$ also found that an overexpression of KDM4A inhibited the differentiation of progenitor cells into osteoblasts in human progenitor cells, and the decreased expression of KDM4A in mouse BMSCs increased the hBMSCs osteogenesis [26].

SB431542, a potent small molecule inhibitor of the TGF- $\beta$ I receptor, was a specific inhibitor of the TGF$\beta /$ Smad signaling pathway [27]. SB431542 had no inhibitory effect on the non-Smad signaling pathway [28]. In this experiment, the expression of KDM4A/4D and methyltransferase EZH1 did not change significantly when hBMSCs were incubated with SB431542 solution. These results suggested that TGF- $\beta 1$ might regulate the expression of KDM4A, KDM4D and EZH1 through non TGF- $\beta /$ Smad 
signaling pathway. Previous studies had proved that EZH1 was related to the non-Smad signaling pathway. It was found that EZH1 regulated the activity of $\mathrm{H} / \mathrm{E}(\mathrm{SPL})$ downstream of the Notch signal pathway through histone methylation, thus regulating epithelial mesenchymal transition [29].

The level of KDM6A/B were both further enhanced by TGF- $\beta 1$ solution stimulation, while the expression of $\mathrm{KDM} 6 \mathrm{~A} / 6 \mathrm{~B}$ was inhibited by SB431542. These results suggested that $\mathrm{KDM} 6 \mathrm{~A} / 6 \mathrm{~B}$ expression were regulated by TGF- $\beta /$ Smad signaling pathway in hBMSCs osteogenic differentiation. The interaction of TGF- $\beta 1$ with KDM6A/6B has also been reported in other literatures. Lu Wang reported that the interaction between the TGF- $\beta 1 /$ Smad signaling pathway and KDM6A was one of the mechanisms of pulmonary fibrosis [30]. Shumei Liang confirmed that KDM6B and TGF- $\beta 1$ in ovarian cancer cells also had a mutual regulatory relationship. The enhanced expression of KDM6B promoted tumor metastasis, which could be attenuated by the TGF- $\beta 1$ blocker reagent [31].

In order to confirm that hBMSCs osteogenesis differentiation was regulated by TGF- $\beta 1$ through controlling KDM6A/6B expressions, we added TGF- $\beta 1$ solution and GSK-J4 solution, an inhibitor of KDM6A/ 6B activity, into the osteogenic medium of hBMSCs. After 72 hours of culture, mRNA and protein levels of Runx2 decreased significantly, which suggested that the expressions of Runx2 were regulated by $\mathrm{KDM} 6 \mathrm{~A} / 6 \mathrm{~B}$, and hBMSCs osteogenic differentiation was regulated by TGF- $\beta 1$ controlling of KDM6A/6B expressions. Tetsunaga T's study results were similar: KDM6B regulated RUNX2 expression and promoted the osteogenic differentiation of hBMSCs. However, KDM6B was not directly linked to the RUNX2 promoter, but it regulated the RUNX2 gene by regulating the BMP and the HOX gene expressions[18]. Hemming $S$ reported that $\mathrm{H} 3 \mathrm{~K} 27 \mathrm{me} 3$ on promoters of RUNX2 and downstream target genes (osteopontin and osteocalcin) was demethylated by KDM6A, resulting in histone demethylation, enhancing the expressions of osteogenic-related-genes such as RUNX2, osteopontin and osteocalcin, ultimately leading to osteogenic differentiation of BMSCs [32].

\section{Conclusion}

In conclusion,the study from above experimental demonstrated that the expressions of demethylase KDM6A/6B were positively regulated by the TGF- $\beta /$ Smad signal transduction pathway, which sequentially up-regulated the expression of RUNX2. The increased expression of RUNX2 promoted the differentiation of hBMSCs into osteoblasts, resulting in subchondral bone osteosclerosis and the occurrence of osteoarthritis. Therefore, these results suggested the epigenetic mechanism of TGF$\beta 1 /$ Smad signal pathway regulating the osteogenic differentiation of hBMSCs which provided a distinct idea for understanding the molecular mechanism of osteoarthritis. However, it remains unclear the expressions of demethylase KDM4A/4D and methylase EZH1 might be regulated by the non TGF- $\beta 1$ Smad signal pathway. Collectively, the future experiments such as KDM6A/6B gene knockout plasmid vitro experiments and animal models in vivo will contribute to a better understand of the detailed informations related to the epigenetic mechanism of TGF- $\beta 1 / \mathrm{Smad}$ signal pathway in osteoarthritis.

\section{Declarations}




\section{Funding}

The study was supported by the researh project from Shiheizi Universitiy in China, project number:ZZZC201717A.

\section{Competing interests}

All authors declare no conflict of interests, including employment, consultancies, stock ownership, honoraria, paid expert testimony, patent applications/registrations, and grants or other funding.

\section{Availability of data and materials}

The datasets used and/or analysed during the current study are available from the corresponding author on reasonable request.

\section{Authors' contributions}

All authors contributed to the study conception and design, more contributions from Jianwei He. Material preparation and data collection were performed by Weiwei Cao and Qinzheng Fang. Analysis were from Liu Wei. The first draft of the manuscript was written by Jianwei He and Inayat Azeem. All authors commented on previous versions of the manuscript, read and approved the final manuscript.

\section{Ethics approval and consent to participate}

The study protocol was approved by the Ethics Committee of the First Affiliated Hospital of Shihezi University, School of Medicine in China. The study was performed in accordance with the ethical standards as laid down in the 1964 Declaration of Helsinki and its later amendments or comparable ethical standards. One subject was ethnic Han Chinese. Written informed consent was taken from the subject.

\section{Consent for publication}

Not applicable

\section{Acknowledgements}

The authors want to thank the staff at the department of orthopedics wards 1 for patient sample collections.

\section{References}

1. S.muraki HO, Yoshimuray TA,N et al (2009) Prevalence of radiographic knee osteoarthritis and its association with knee pain in the elderly of Japanese population-based cohorts: the ROAD study. Osteoarthr Cartil 17(9):13 
2. Meina JS, Di Chen HJW, Hee-Jeong et al (2011) Recent progress in understanding molecular mechanisms of cartilage degeneration during osteoarthritis. Ann N Y Acad Sci 1240(12):10

3. Xu Tang SW, Siyan Zhan and Jianhao Lin et al, The Prevalence of Symptomatic Knee Osteoarthritis in China results From the China Health and Retirement Longitudinal Study. ARTHRITIS \& RHEUMATOLOGY, 2016. 68(3): p 654

4. Focht BC, How Knee Osteoarthritis Patients Can Use Exercise to Enhance Quality of Life. ACSM's HEALTH \& FITNESS JOURNALA. 16(5): p. 29

5. Hendon JH, Apazidis DS (2001) A, Recent socioeconomic trends in orthopaedic practice. J Bone Joint Surg Am 83-A(7):1097-1105

6. Liang Xie FT, Wang X, Li F, Cao Xu et al, Systemic neutralization of TGF- $\beta$ attenuates osteoarthritis. Annals of the New York Academy of Sciences, 2016. 1376(1): p 63

7. Suri SW (2012) D.A, Osteochondral alterations in osteoarhtritis. Bone 51(2):212

8. David J, Hunter YZ, Niu J, Felson DT (2006) Increase in bone marrow lesions associated with cartilage loss:a longitudinal magnetic resonance imaghing study of knee osteoarthritis. Arthritis rheumatism 5(54):1536

9. Gehua Zhen CW, Jia X, Cao Xu,et al (2013) Inbition of TGF-beta signaling in mesenchymal stem cells of subchondral bone attenuates osteoarthritis. Nat Med 19(6):713

10. Allis CD, Cote BS, Dent J, Jenuwien S, Kouzarides T (2007) T, et al, New nomenclature for chromatinmodifying enzymes. Cell 131(4):637

11. A.Bird, Perceptions of epigenetics Nature, 2007. 447(7143): p. 399

12. Gibney ER (2010) C.M.N., Epigenetics and gene expression. Heredity 105(1):13

13. Silvia Monteagudo FMFC, Aznar-Lopez C, Yibmantasiri P, Guns L-A,Peter Carmeliet, Fre' de'ric C, Lories RJ, DOT1L safeguards cartilage homestasis and protecets against osteoarthritis. Nat Commun (2017) 19(8)

14. Vo Linda T (2018) K.M.A., Xin Liu and George Q Daley etal, Regulation of embryonic haematopoietic multipotency by EZH1. Nature 553(7689):510

15. Rodova M 1, Li QL,Y, Wang J et al, Nfat1 Regulates Adult Articular Chondrocyte Function through Its Age-Dependent Expression Mediated by Epigenetic Histone Methylation. J Bone Miner Res, 2011. 26(8): p. 1987

16. Cyrille C.Thinnes KSE, Akane Kawamura, Richard J (2014) Hopkinson etal, Targeting histone lysine demethylases - Progress, challenges and the future. Biochem Biophys Acta 1839(5):1433

17. Aukasz A, Poniatowski P, Gasik R, Szukiewicz andDariusz, Transforming Growth Factor Beta Family: Insight into the Role of Growth Factors in Regulation of Fracture Healing Biology and Potential Clinical Applications. Mediators of Inflammatio, 2015. 2015(137823): p 17

18. Ling Ye ZF, Yu B, Chang J, Hezaimi KA, Zhou X, NoHee Park, Wang C-Y, Histone demethylases KDM4B and KDM6B promotes osteogenic differentiation of Human MSCs. Cell Stem Cell (2012) 11(1): p. 12 
19. Lee H-L, Deng BY,P, Honga $C$ et al (2016) Transforming growth factor-b -induced KDM4B promotes chondrogenic differentiation of human mesenchymal stem cell. Stem Cells 34(3):720

20. Anna Cascante SK, Biswas M, Hermanson O et al, Gene-Specific Methylation Control of H3K9 and H3K36 on Neurotrophic BDNF versus Astroglial GFAP Genes by KDM4A/C Regulates Neural Stem Cell Differentiation. J. Mol. Biol, 2014. 4c(4): p. 12

21. Rui Dong RY, Du J, Fan Z (2013) Depletion of histone demethylase KDM2A enhanced the adipogenic and chondrogenic differentiation potentials of stem cells from apical papilla. Exp Cell Res 319(18):2882

22. Chen X, Zhang ZF,S (2018) Spatial and temporal expression of histone demethylase KDM2B in the development of molar and mandible in mice. Beijing Journal of Stomatology 26(5):245

23. Dudakovic A, Camilleri ET, Xu F, Riester SM, McGee-Lawrence ME, Bradley EW, van Wijnen AJ, et at, Epigenetic control of skeletal development by the histone methyltransferase Ezh2. Journal of Biological Chemistry (2015) 290(46): p. 14

24. Dominici M (2006) K.L.B., and Em Horwitz, etal, Minimal critetia for defining multipotent messenchymal stromal cells. The international society for cellular therapy position statement. Cytotherapy 8(4):335

25. Pittenger MF et al (1999) Multilineage potential of adult human mesenchymal stem cells. Science 284(5411):147

26. Qi Qi YW, Xiaochen, Wang, Baoli, Wang et al (2020) Histone demethylase KDM4A regulates adipogenic and osteogenic differentiation via epigenetic regulation of C/EBPa and canonical Wnt signaling. Cell Mol Life Sci 77(8):2422

27. Chang MC, Wu PY HL and Jeng JH et al (2019) Stimulation of MMP-9 of oral epithelial cells by areca nut extract is related to TGF- $\beta$ /Smad2-dependent and -independent pathways and prevented by betel leaf extract, hydroxychavicol and melatonin. Aging 11(23):11640

28. Shi A, Bao HA D and Qin H et al (2019) Small molecule ihibitor of TGF- $\beta$ signaling enables robust osteogenesisi of autologous GMSCs to successfully repair minipig severe maxillofacial bone defects. Stem Cell Res Ther 10(1):10

29. Miroslav Blumenberg SG, Dickman K, Zavadil J et al (2007) Chromatin structure regulation in transforming growth factor-beta-directed epithelial-mesenchymal transition. Cells Tissues Organs 185(1-3):175

30. Lu Wang JL, Xu B et al (2019) miR-425 reduction causes aberrant proliferation and collagen synthesis through modulating TGF- $\beta$ /Smad signaling in acute respiratory distress syndrome. Int $\mathrm{J}$ Clin Exp Pathol 12(7):2613

31. Shumei Liang QY, Wang Yun-shan et al KDM6B promotes ovarian cancer cell migration and invasion by induced transforming growth factor- $\beta 1$ expression. J Cell Biochem (2018) 58(7): p. 14

32. Hemming S, C.D., and Gronthos S (2014) etal, EZH2 and KDM6A act as an epigenetic switch to regulate mesenchymal stem cell lineage specification. stem Cells 32(3):816 


\section{Tables}

Table 1 The primers used for the qRT-PCR in this research were sequenced as below

Target gene Primer 5'-3'

\begin{tabular}{lll}
\cline { 2 - 2 } & Forward & Reverse \\
\hline$R U N X 2$ & gtggacgaggcaagagtttca & catcaagcttct gtctgtgcc. \\
\hline PPARY & CCAGAAAGCGATTCCTTCAC & CACG TTAGTTTCACCTCGGA. \\
\hline SOX9 & GGAGATGAAATCTGTTCTGGGAAT & TGAAGGTTAACTGCTGGTGTTCTG \\
\hline$K D M 2 A$ & CCAAAGGTGCGGGTTCCTAC & GGCTCCTGACACAATCGGG \\
\hline$K D M 2 B$ & CTCACTGCTGTTGGCACCAC & TGCTTGCAGTACCTCAGGTCAATA \\
\hline$K D M 4 A$ & GGGCTTTGGGCTGTAGATTC & GCTCGTGGCTTCCACTCTTT \\
\hline$K D M 4 B$ & CCCTGCGGTGGATTGACTAC & GTTTCCGGTGAGACCTGCG \\
\hline$K D M 4 C$ & AAGCTCGATTTTCCACAGCC & CAGGGTCGGCCACATATTCA \\
\hline$K D M 4 D$ & GGGCAGGGGTGTTTACTCAAT & TGTTTGCCAAATGGCGATACT \\
\hline$K D M 6 A$ & GCCTCTTTGGGTTCGTGAGAT & AGGCAGCATTCTTCCAGTAGT \\
\hline$K D M 6 B$ & CTGAACTTGGGCCTGTTGTC & GCCTGTCAGGTCCCAGTTCT \\
\hline$E Z H 1$ & AATATGGGAGCAAAGGCTCTGTATGTG & CACGAAGTTTCTTCCACTCTTCATTGAG \\
\hline$E Z H 2$ & TACTTGTGGAGCCGCTGAC & TCTGCCACGTCAGATGGTG \\
\hline$R U N X 2$ & gtggacgaggcaagagtttca & catcaagcttctgtctgtgcC \\
\hline$G A P D H$ & CGGACCAATACGACCAAATCCG & AGCCACATCGCTCAGACACC
\end{tabular}

Table 2 The first antibody dilution ratioes in the western blot tests were listed 
Antibodies' names

Rabbit Anti-Human RUNX2

Rabbit Anti-Human KDM4A

Rabbit Anti-Human KDM4D

Rabbit Anti-Human EZH1

Rabbit Anti-Human KDM6A

Rabbit Anti-Human KDM6B

Rabbit Anti-Human GAPDH

HRP-labeled goat anti-rabbit secondary antibody
Company(lot) $\square$ Dilution ratio[

Abcam/(ab23981)『WB 1ه1000)

Abcam/(ab191433)凶WB 1ه1000)

Abcam/(ab93694)凶WB 1ه1000)

Abcam/(137693) هWB 1ه1000)

Abcam/ab84190『WB 1ه1000》

Abcam/ab169197هWB 1ه1000》

Proteintech /10494-1-AP『WB 1ه10000区

Proteintech/SA00004-2『WB 1囚500区

\section{Figures}


A

$2 \mathrm{~d}$

$4 \mathrm{~d}$

8 d

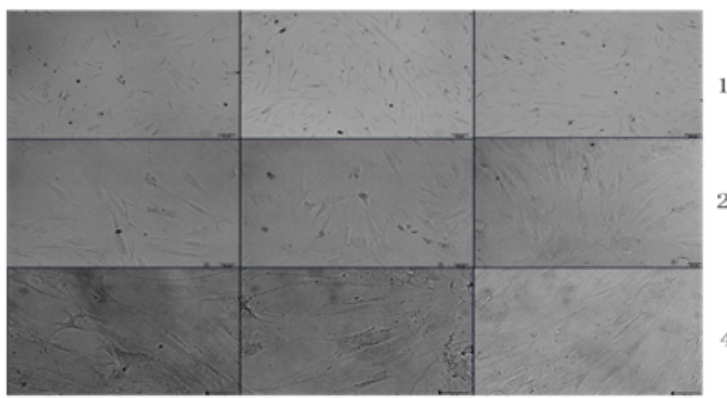

$100 \times$

$200 \times$

$400 \times$

B
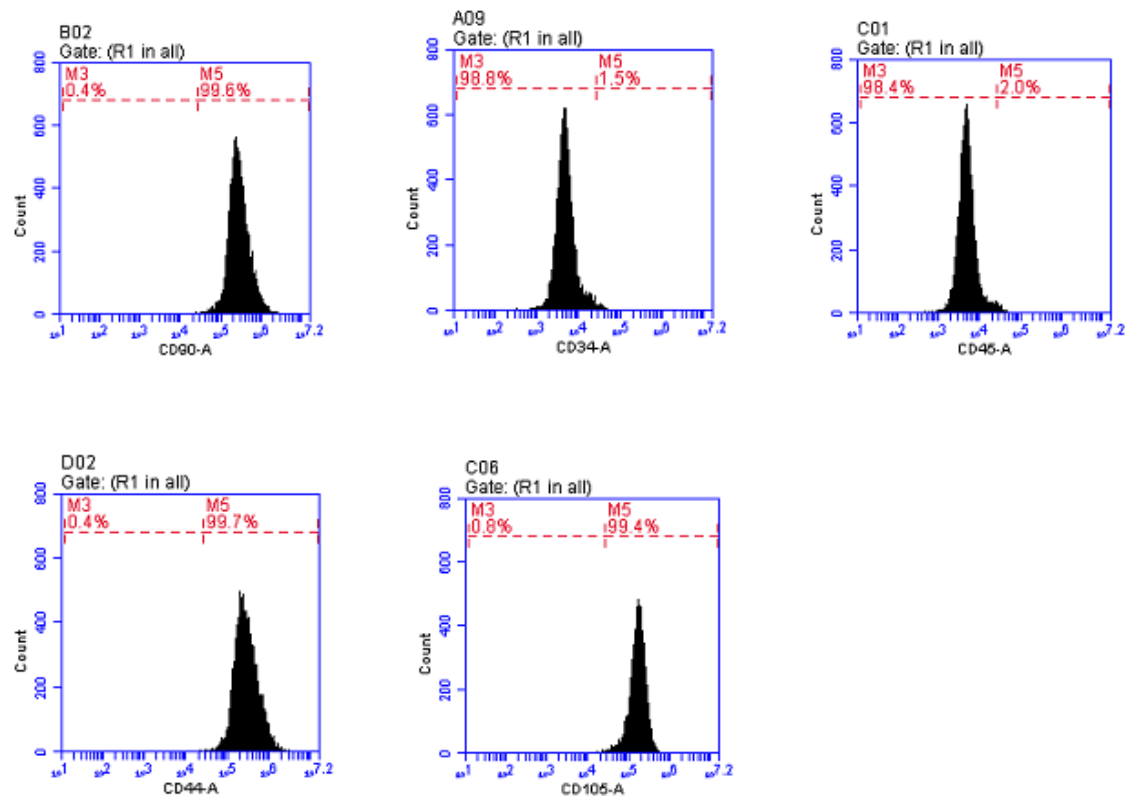

C.

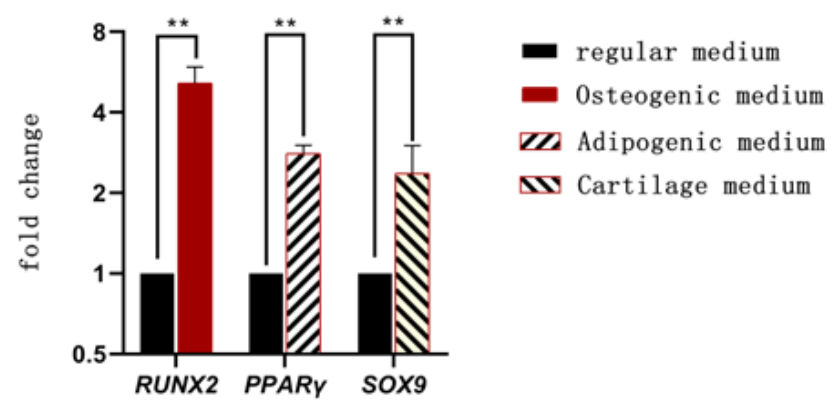

Figure 1

hBMSCs cultured in regular medium was identified by flow cytometry tests, and the multi-diffenentiation potential quality was confirmed by qRT-PCR assays through detecting RUNX2, PPARY and SOX9 mRNA levels in related cell culture mediums. A. hBMSCs were cultured and adhered for 2/4/8 days in the regular stem cell culture medium. B. antigen expressions on hBMSCs surface were detected by flow cytometry after 8-days culture. CD34, CD45, CD44, CD90 and CD105 were shown in panels from left to right, from 
top to bottom. C. The expressions of RUNX2, SOX9 and PPARY mRNA were showed all increasement in hBMSCs after 6-day culture in differentiation culture medium. All mRNA expression levels was standardized with GAPDH, and the standard deviation came from 3 independent experiments $n=3$. (** means $\mathrm{P}<0.01)$.

Bone alkaline phosphatase staining

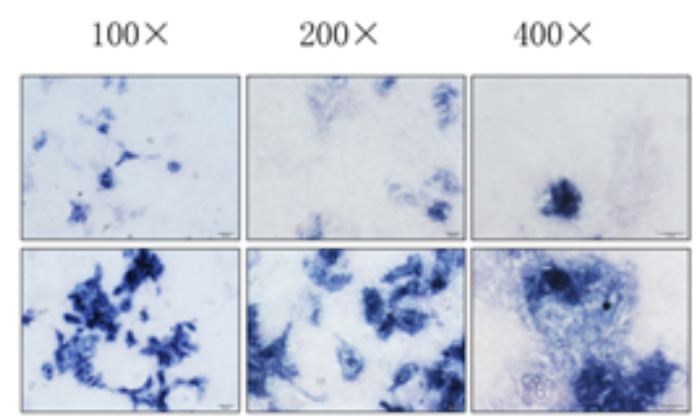

6-well plate

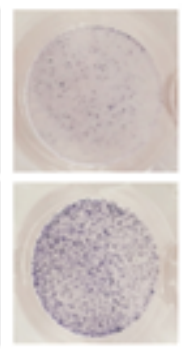

Osteogenic induction culture 6 days

Osteogenic induction and TGF- $\beta 1$

culture 6 days

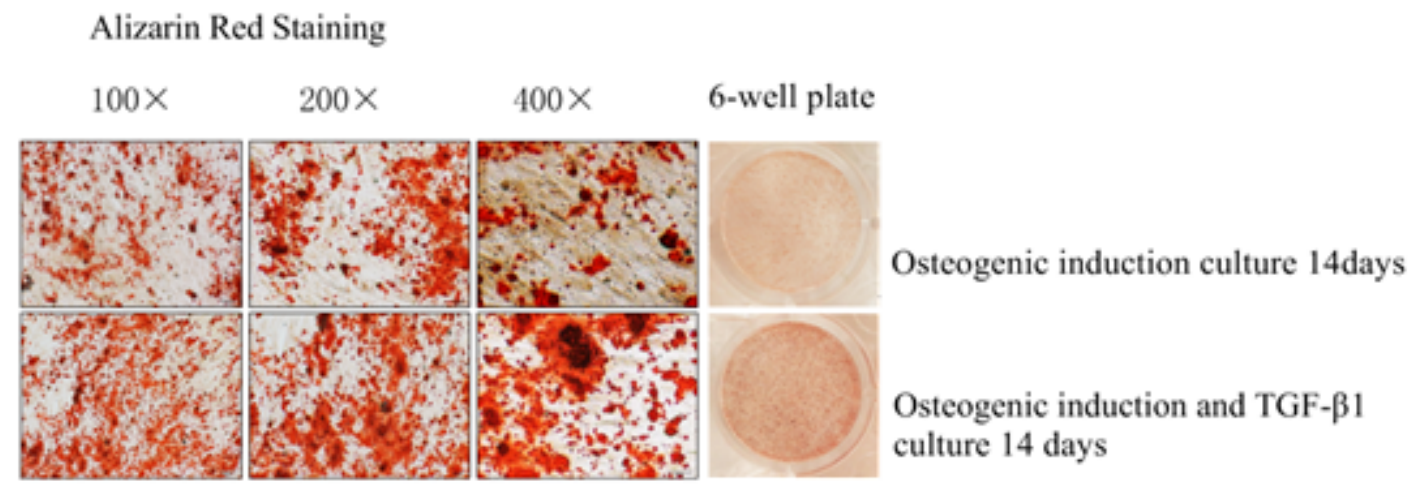

hBMSCs used osteogenic culture + TGF- $\beta 1$ group, compared with osteogenic culture group, osteoalkaline and alizarin staining were significantly enhanced

\section{Figure 2}

TGF- $\beta 1$ promotion effects on the hBMSCs' differentiation into osteoblasts was showed by bone alkaline phosphatase and alizarin red staining. The hBMSCs were devided into two groups, one was the osteogenic medium group, the other was osteogenic medium incubated with TGF- $\beta 1$ (10ng/mL). TGF- $\beta 1$ group staining was more darker. 


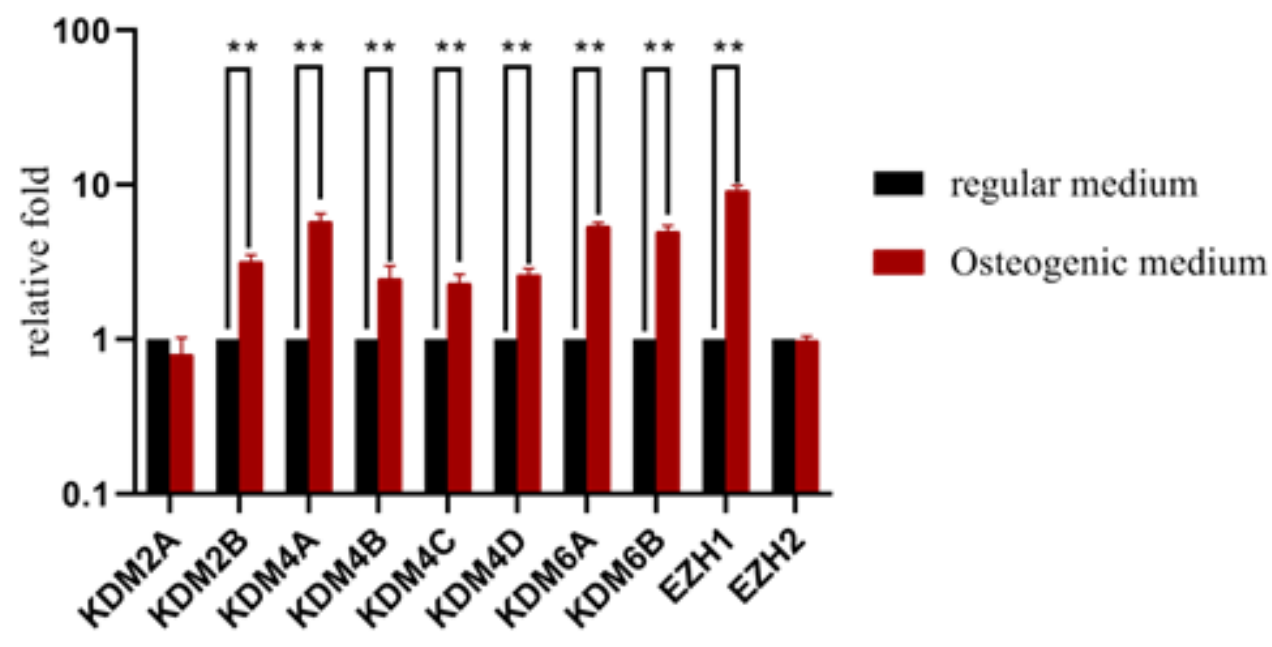

Realtime PCR detection of mRNA expressions of related methylase and demethylase before and after BMSCs osteogenic differentiation

\section{Figure 3}

The mRNA levels of some methyltransferase and demethylase in hBMSCs were improved after hBMSCs were cultured in osteogenic medium for 3 days. qRT-PCR assay was used to detect the mRNA expressions and the mRNA expression was standardized with GAPDH. The standard deviation came from three independent experiments with 3 replicates for each experiment. (** means $P<0.01, n=3$ ) 


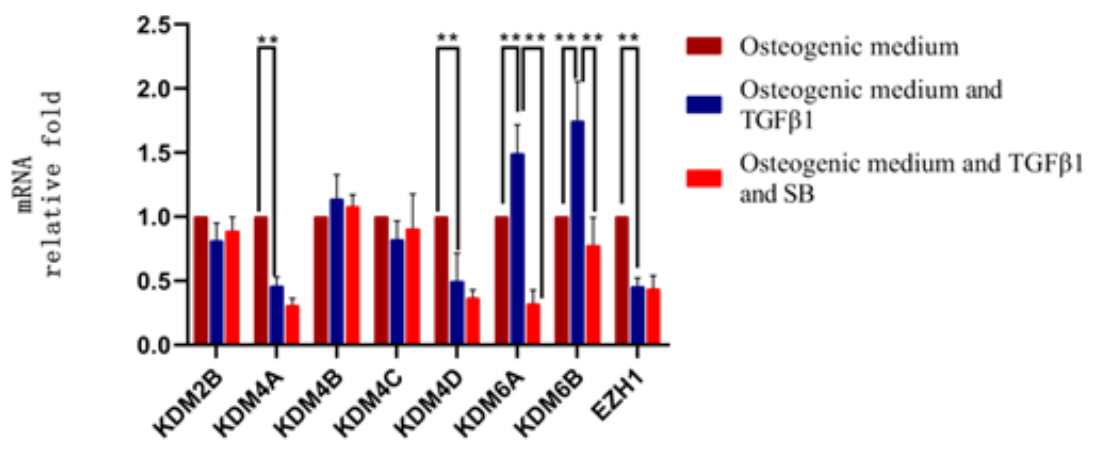

TGF- $\beta 1$ regulated mRNA expression of methyltransferase and demethylase during TGF- $\beta 1$ promoted the osteogenic differentiation of hBMSCs

B
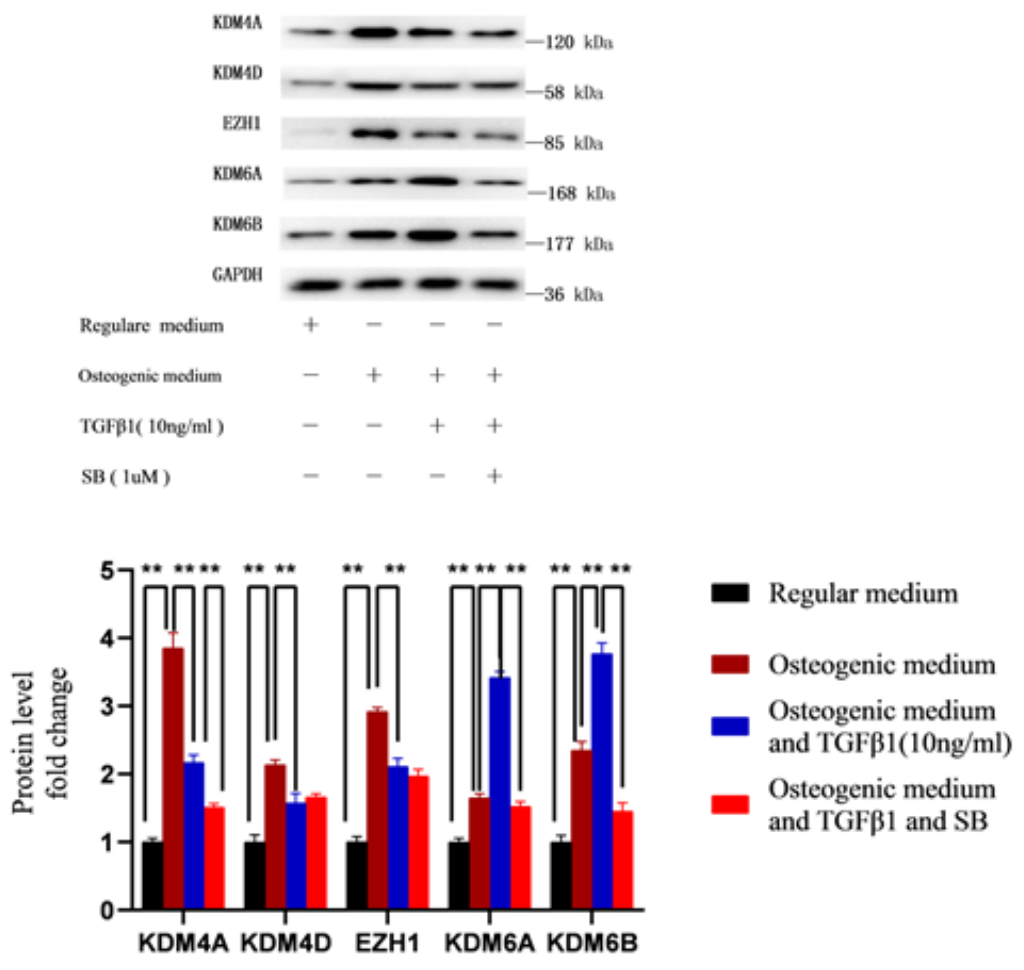

Methyltransferases and demethylases protein expression levels were regulated by TGF- $\beta 1$ in the process of promoting the hBMSCs osteogenic differentiation

\section{Figure 4}

The expressions of methyltransferase EZH1 and demethylase KDM4A/4D and KDM6A/6B were regulated by TGF- $\beta 1$. Negative regulation was observed in EZH1, KDM4A and KDM4D, while positive regulation was KDM6A/6B expressions. A. After hBMSCs were cultured for $72 \mathrm{hrs}$, the mRNA expressions of methyltransferase and demethylase in each group were detected by qRT-PCR, and the mRNA expression was then standardized by GAPDH. The mRNA expression level in the osteogenic medium group was 
taken as 1 , (** means $P<0.01, n=3)$ B. Protein level of demethylase and methyltransferases were tested by Western Blot method. All protein expression levels were standardized by GAPDH and the conventional culture histone expression level was 1 , and the relative protein expression level was calculated. ( ${ }^{\star \star}$ means $\mathrm{P}<0.01, \mathrm{n}=3)$.

$\mathbf{A}$

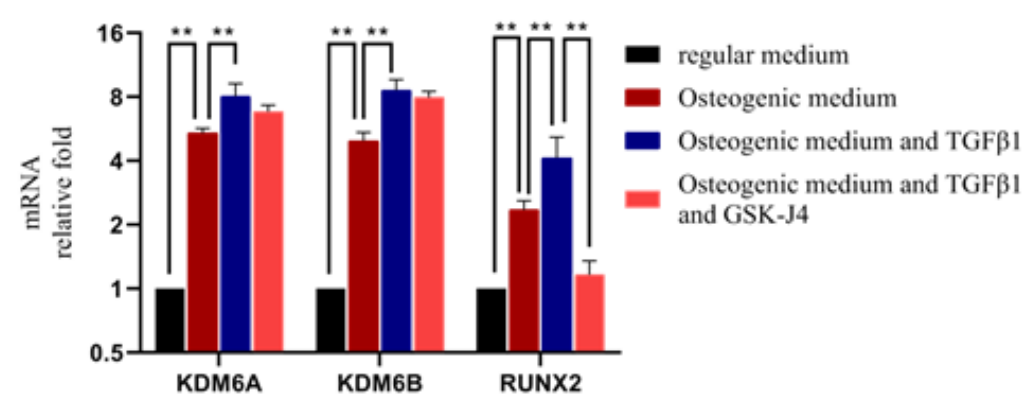

KDM6A and KDM6B positively regulated mRNA

expression of osteogenic transcription factor RUNX2.

B
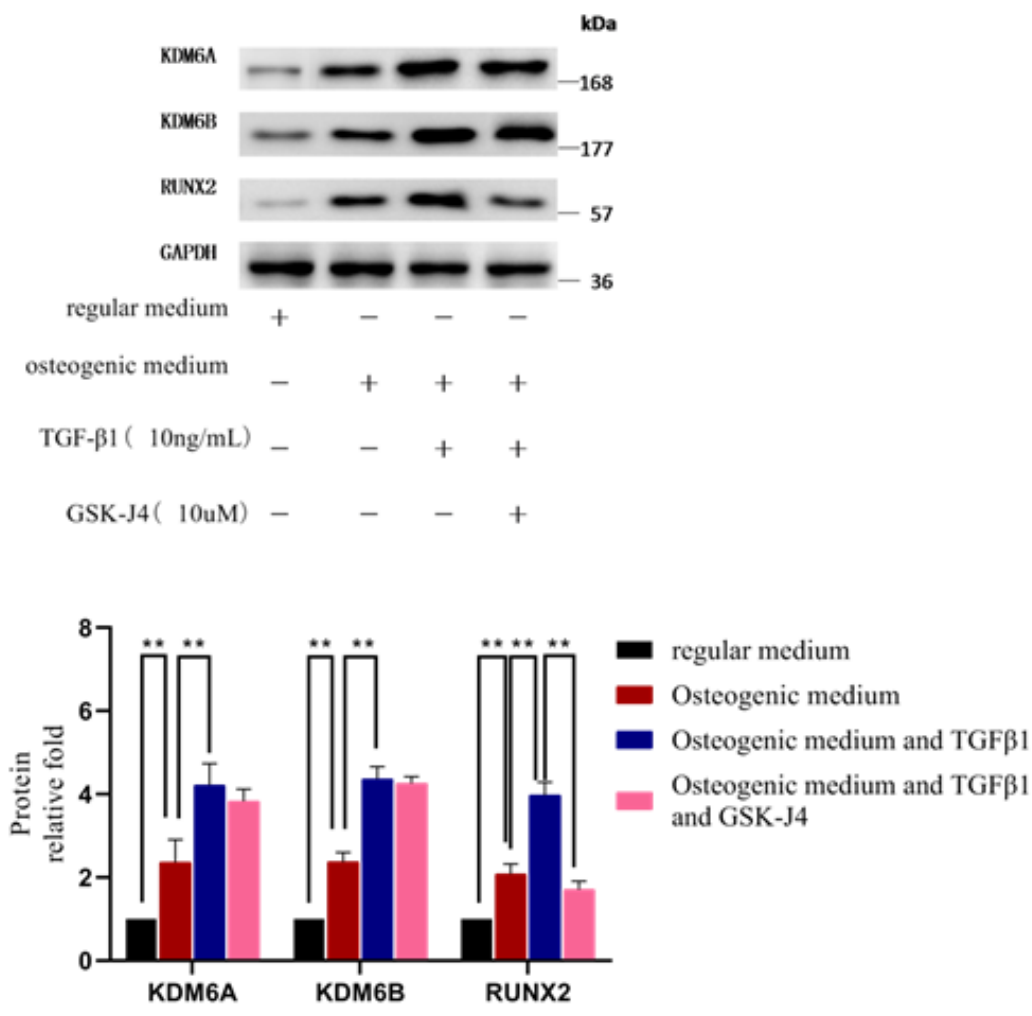

Regulatory effects of demethylase KDM6A and KDM6B on the protein level of osteogenic transcription factor RUNX2

Figure 5 
The expression of the osteogenic transcription factor RUNX2 was positively regulated by KDM6A/6B. A. The mRNA level was assayed by qRT-PCR method detecting the mRNA expressions of KDM6A, KDM6B and RUNX2. All mRNA expression levels were standardized to GAPDH. The mRNA expression level of each index in the conventional culture group was 1 . the relative expression of mRNA in each group was calculated. $\nabla^{\star \star}$ means $P<0.01, n=3 \otimes B$. The protein expressions of the KDM6A/6B and RUNX2 were assayed by Western Blot. All protein expression levels were standardized with GAPDH, and the relative expression levels of each protein were calculated according to the regular culture medium group, and the regular culture group as 1 . ( ${ }^{\star \star}$ means $\mathrm{P}<0.01, \mathrm{n}=3$.) 